\title{
Número y Disposición de los Forámenes Apicales en la Superficie Radicular de Primeros Premolares Superiores
}

\author{
Number and Arrangement of the Apical Foramen \\ in Root Surface of Maxillary First Premolars
}

Ludovic Doussoulin G.","; Constanza Galleguillos V."* \& Constanza Gálvez B."*

DOUSSOULIN, G. L.; GALLEGUILLOS, V. C.; GÁLVEZ, B. C. Número y disposición de los forámenes apicales en la superficie radicular de primeros premolares superiores. Int. J. Odontostomat., 10(3):419-424, 2016.

RESUMEN: El primer premolar maxilar es considerado un diente con alta variación en su anatomía radicular y morfología de canales radiculares (Chaparro et al., 1999). Por esto, toma gran relevancia el conocimiento de su anatomía al realizar tratamientos endodónticos. El objetivo de éste estudio fue describir frecuencia de número y disposición de forámenes apicales en la región apical radicular en primeros premolares maxilares. Se utilizó microscopia óptica para estudiar en detalle la anatomía radicular apical de 141 raíces de primeros premolares maxilares unirradiculares $(n=51)$ y birradiculares $(n=49)$ de nacionalidad chilena; en donde se observó número de forámenes apicales mayores y accesorios (foraminas), ubicación del foramen mayor, y distancia de éste al ápice anatómico. En dientes unirradiculares se encontró una mayor frecuencia de uno o dos forámenes mayores, y un promedio de 2,5 foraminas accesorias por diente analizado. En dientes birradiculares se encontró una mayor frecuencia de un foramen mayor por raíz, y un promedio de 1,3 foraminas por raíz. Además, se encontró que la ubicación del foramen mayor fue más frecuente en las superficies mesial-distalpalatino en dientes unirradiculares, no encontrando diferencias significativas entre éstas superficies. En cambio en dientes birradiculares se observó que el foramen apical mayor en todas las superficies radiculares sin haber diferencias significativas entre ellas. La distancia promedio entre el foramen mayor y el ápice anatómico fue de 0,819 mm en uni y birradiculares. En conclusión los primeros premolares maxilares representativos de población chilena, presentan una anatomía apical variable y compleja, en cuanto al número de forámenes mayores, número de foraminas, y su ubicación.

PALABRAS CLAVE: frecuencia foramen apical mayor, frecuencia foraminas accesorias, distancia foramen apical al ápice, superficie radicular primer premolar maxilar.

\section{INTRODUCCIÓN}

El objetivo de la terapia endodóntica es la eliminación de tejido pulpar o tejido necrótico y microorganismos, desbridación del sistema de canales radiculares y su conformación para la obturación tridimensional de estos, recuperando o manteniendo la salud de los tejidos periapicales (Kuttler, 1958).

Para obtener un tratamiento endodóntico exitoso, se requiere del conocimiento tanto de la morfología normal, como de las variaciones anatómicas de los dientes.

El estudio de la anatomía siempre ha sido un tema relevante en investigación, y la anatomía endodóntica no es ajena a esto, ya que, existe una gran variabilidad en la disposición del sistema de canales radiculares. El foramen apical presenta una topografía muy particular, y no en continuidad con el eje mayor del conducto principal. (Ferlini-Filho, 1999).

Morfológicamente, se observa una gran variabilidad en la distancia entre el ápice radiográfico y la salida del foramen apical. Además se ha demostrado que es muy común que presenten ramificaciones en el área apical (Gutiérrez \& Aguayo, 1995; Levy \& Glatt, 1998). Por lo que, tener el conocimiento acabado de la morfología sin duda ayudará a realizar un tratamiento

* Profesor Asociado, Facultad de Ciencias, Escuela de Odontología, Universidad Mayor, Santiago, Chile.

* Cirujano Dentista, Especialista en Endodoncia, Magíster en Pedagogía Universitaria, Santiago, Chile.

*** Cirujano Dentista egresada Universidad Mayor, Facultad de Odontología, Universidad Mayor, Santiago Chile. 
endodóntico lo más conservador posible, para lograr los objetivos del tratamiento, y así también disminuir factores que puedan llevar a provocar accidentes clínicos.

El primer premolar maxilar es considerado un diente con gran variación en su anatomía radicular y morfología de canales radiculares. Esta variación en número y tipo de canales radiculares es lo más descrito como una variante anatómica en la literatura. La presencia de dos canales se considera como normal, aun cuando puedan existir diferencias raciales; como el número de forámenes principales y accesorios, la desviación del foramen apical mayor de su ápice anatómico y la superficie en donde éste se ubica (Chaparro et al.). Debido a esto, es de gran relevancia generar un conocimiento detallado de la anatomía de éste diente en específico, ya que, es un diente con una alta incidencia de caries que en la mayoría de los casos compromete también el órgano pulpar, lo cual deriva en serias consecuencias fisiopatológicas y clínicas. Además, lo anterior genera otras situaciones en donde se debe realizar tratamiento endodóntico por razones protésicas y/o periodontales.

La anatomía endodóntica es un tema de interés para la investigación mundial. A pesar de su relevancia, en Chile no se han realizado estudios en este grupo dentario.

\section{MATERIAL Y MÉTODO}

Se estudió la anatomía radicular apical de 100 primeros premolares superiores en pacientes de nacionalidad chilena; 51 unirradiculares y 49 birradiculares, en donde se obtuvo un total de 141 raíces estudiadas. Las muestras fueron obtenidas de consultorios públicos, clínica odontológica de la Universidad Mayor y clínicas privadas de la Región Metropolitana. Las muestras corresponden a dientes recientemente extraídos, ya sea por: causas periodontales, ortodóncicas, protésicas y/o afectados por caries, con ápice cerrado y con su superficie radicular indemne. Como criterio de exclusión, se descartaron de este estudio aquellos dientes: (1) extraídos con corona irreconocible que no permitían la identificación clara de sus caras dentarias, (2) dientes con fractura radicular, (3) dientes con reabsorción radicular apical, (4) dientes con foramen apical mayor manipulado endodónticamente (sobreinstrumentado y/o sobreobturado).
Todas las muestras fueron desbridadas con una cureta Gracey número 5-6 para eliminar cálculo y restos de tejido periodontal adheridos, posteriormente se sumergieron en hipoclorito de sodio al 5,25\% durante 1 hora para eliminar posibles restos de materia orgánica y luego fueron conservados en suero fisiológico. Los dientes birradiculares fueron seccionados con un disco carburunbum en la zona de la furca, para poder tener una observación completa de la superficie radicular apical. A continuación se colocó cada muestra en un cubo de silicona pesada, el cuál previamente tenía identificada cada superficie radicular; dejando el tercio apical expuesto para su estudio.

Las muestras fueron observadas en la totalidad de su superficie radicular apical (vestibular, mesial, palatina, distal y apical); identificando el número de forámenes apicales mayores y accesorios, además de la superficie en donde éste se encuentra observado bajo el microscopio Leica ICC 50 HD con cámara integrada, y objetivo de $4 x$. Se tomaron microfotografías y se analizaron con el software LAS EZ®, con el cual se midieron las distancias existentes entre el foramen apical mayor y el ápice anatómico, mediante un trazado desde, el punto superior del foramen apical mayor hasta el punto superior del vértice anatómico. Los trazados obtenidos posteriormente se llevaron a barras de escala del software, con las que se obtuvieron las medidas en milímetros de la distancia descrita.

Para cuantificar el número de forámenes apicales principales presentes en la superficie radicular, se consideraron como tales, aquellos que presentaron un diámetro igual o mayor a 0,10 milímetros. Todo aquel que midió menos de $0,10 \mathrm{~mm}$ fue considerado como foramen apical accesorio o foramina. (Marroquin et al., 2004).

Los resultados obtenidos fueron registrados y tabulados en una planilla Exce ${ }^{\circledR}$, para el posterior análisis estadístico, el cual se realizó con el software Stata ${ }^{\circledR}$ mediante el test Student en base a un promedio conocido y chi cuadrado. Con los datos obtenidos se estableció una frecuencia del número y disposición de los forámenes apicales estudiados, y se determinó un promedio de la distancia del foramen apical mayor al ápice anatómico.

Para tener una mayor fiabilidad en los resultados, se realizó una calibración del observador. Ésta se obtuvo observando 10 muestras escogidas al azar, en las cuales se debieron observar los parámetros anatómicos establecidos. Una semana después se repitió 
el procedimiento. Así se pudo comparar los resultados obtenidos, para ver el grado de fiabilidad del observador.

\section{RESULTADOS}

\section{Frecuencia foramen apical mayor en primer premolar superior}

Dientes unirradiculares. Una mayor frecuencia de encontrar uno $(44,07 \%)$ o dos forámenes apicales mayores $(30,51 \%)$ en la superficie radicular apical de primeros premolares maxilares unirradiculares, no existiendo una diferencia significativa entre éstas $(p=0,228)$. $Y$ una menor frecuencia de encontrar tres, cuatro y cinco forámenes $(15,25 \%)$ en la superficie radicular de primeros premolares superiores. Estableciéndose una diferencia significativa entre encontrar uno o dos forámenes comparado a encontrar tres, cuatro o cinco $(p 1=0,004)(p 2=0,0005)$ y $(p 3=0,0005)$. Tabla I.

Tabla I. Frecuencia del número de forámenes apicales mayores en dientes uni y birradiculares.

\begin{tabular}{lcccc}
\hline & \multicolumn{2}{c}{ Unirradiculares $(\mathbf{n}=\mathbf{1 1 5})$} & \multicolumn{2}{c}{ Birradiculares $(\mathbf{n = 1 0 6})$} \\
$\mathbf{N}^{\mathbf{0}}$ FAM & Frecuencia $(\mathrm{n})$ & Porcentaje $(\%)$ & Frecuencia $(\mathrm{n})$ & Porcentaje $(\%)$ \\
\hline 1 & 26 & 44,07 & 62 & 75,61 \\
2 & 18 & 30,51 & 16 & 19,51 \\
3 & 9 & 15,25 & 4 & 4,88 \\
4 & 4 & 6,78 & 0 & 0,00 \\
5 & 2 & 3,39 & 0 & 0,00 \\
Total & 59 & 100.00 & 82 & 100,00 \\
\hline
\end{tabular}

NoFAM= Número de foramen apical mayor. $(n)=$ Número de raíz.

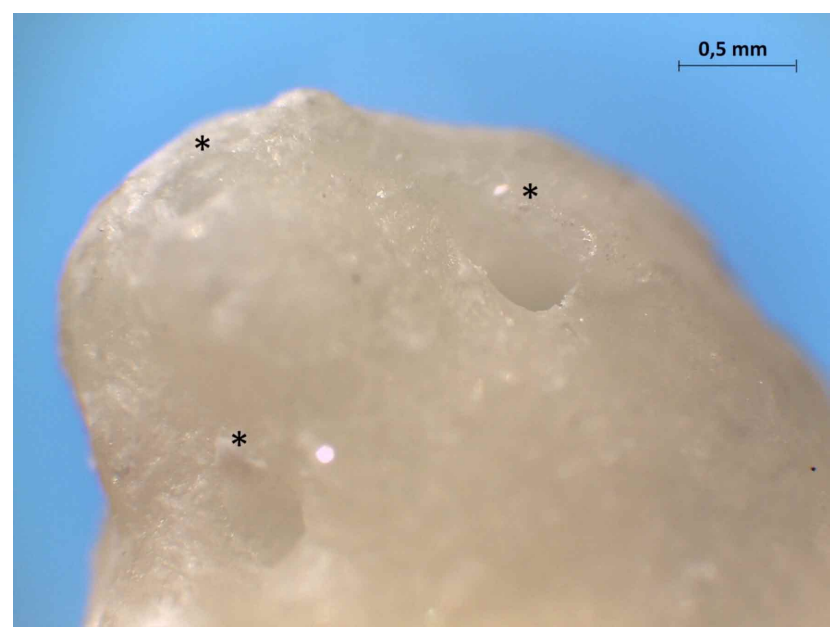

Fig. 1. Microfotografía aumento 4x, forámenes apicales mayores en superficie apical primer premolar superior unirradicular. $\left(^{*}\right)$ Forámenes apicales mayores. un $9,22 \%$.
El promedio del número de forámenes presentes en la superficie radicular apical de primeros premolares superiores unirradiculares fue de 1,94 forámenes. Fig. 1.

Dientes birradiculares. Se observó que hubo una mayor frecuencia de encontrar un foramen apical mayor en la superficie radicular apical de primeros premolares maxilares birradiculares $(75,61 \%)$, existiendo una diferencia significativa $(p<0,005)$ comparado con la emergencia de dos $(19,51 \%)$ o tres forámenes $(4,88$ $\%)$. Pero no existió diferencia significativa entre encontrar dos o tres forámenes por raíz en la superficie radicular de primeros premolares superiores $(p 1>0,005)(p 2>0,005)$.

Al comparar la frecuencia de forámenes apicales mayores entre raíz vestibular y raíz palatina, se observó que lo más predominante fue la presencia de un foramen por raíz; con un 65,85 \% en la raíz vestibular y un $85,37 \%$ en la raíz palatina. No hubo una diferencia significativa en el número de forámenes apicales mayores entre las raíces vestibular y palatina respectivamente. $\quad(p 1=0,310)$ $(p 2=0,34)(p 3=0,317)$. Tabla I.

El promedio del número de forámenes presentes en la superficie radicular apical de primeros premolares superiores birradiculares fue de 1,41 forámenes en la raíz vestibular y 1,17 forámenes en la raíz palatina.

Totalidad de las muestras. En la totalidad de las raíces observadas (141), el porcentaje del número de los forámenes apicales mayores por raíz, fue de un foramen por raíz en un $62,41 \%$, de dos forámenes por raíz $24,11 \%$ y de tres forámenes por raíz tan solo

\section{Frecuencia forámenes apicales accesorios en pri- mer premolar superior (foraminas)}

Dientes unirradiculares. Se observó que un 27,12 $\%$ no presentó foraminas accesorias, un $15,25 \%$ presentó cuatro foraminas, seguido por la presencia de una y dos foraminas, ambas con un $13,56 \%$, un 11,86 $\%$ presentó 3 y 5 foraminas, posteriormente se observó una disminución de la frecuencia de éstas, llegando a observarse hasta 11 foraminas con un 1,69\%. 
El promedio de foraminas presentes en la superficie radicular apical fue de 2,5 foraminas.

Dientes birradiculares. Se obtuvo que un $62,20 \%$ de las raíces observadas no presentó foraminas accesorias observables, y en un 10,98\% presentó dos foraminas, seguido por la presencia de una foramina con un valor de $8,54 \%$, un $4,88 \%$ presentó 4 foraminas, posteriormente se observó una disminución de la frecuencia de éstas, llegando a observarse hasta 10 foraminas con un $2,44 \%$.

El promedio de foraminas presentes en la superficie radicular apical fue de 1,3 foraminas por raíz. Frecuencia de ubicación de foramen apical mayor

Dientes unirradiculares. Se encontraron diferencias significativas en la probabilidad de emergencia del foramen entre las superficies radiculares apicales $(p=0,0005)$. Siendo mayor la frecuencia en las superficies mesiales $(32,17 \%)$, distales $(29,56 \%)$ y palatinas $(22,60 \%)$, sin existir una diferencia significativa entre éstas $(p=0,368)$. Comparado con las superficies vestibulares y apicales, en las que se observó la emergencia del foramen apical en una menor frecuencia, en donde si habrá diferencias significativas $(p 1=0,0005)(p 2=0,004)$. Tabla II.

Dientes birradiculares. Se observó que la ubicación más frecuente del foramen apical mayor en ambas raíces fue en la superficie palatina, en la raíz vestibular $27,55 \%$ y con un $27,09 \%$ en la raíz palatina, seguido por la superficie distal con un 24,24 $\%$ en la raíz vestibular y un $22,92 \%$ en la raíz palatina. Sin embargo, no hubo diferencias significativas en la probabilidad de emergencia del foramen entre las

cinco superficies radiculares apicales tanto de la raíz vestibular $(p 1=0,127)$, como en la raíz palatina $(\mathrm{p} 2=0,551)$. Tabla II. apical en primeros premolares superiores uni y birradiculares.

\begin{tabular}{lllllll}
\hline & \multicolumn{2}{l}{ Unirradiculares } & \multicolumn{2}{c}{$\begin{array}{l}\text { Birradiculares } \\
\text { Raíz vestibular }\end{array}$} & \multicolumn{2}{c}{ Raíz Palatina } \\
Superficie Radicular & $\mathrm{F}(\mathrm{n})$ & $(\%)$ & $\mathrm{F}(\mathrm{n})$ & $(\%)$ & $\mathrm{F}(\mathrm{n})$ & $(\%)$ \\
\hline Vestibular & 9 & 7,8 & 12 & 20,7 & 8 & 16,7 \\
Mesial & 37 & 32,2 & 12 & 20,7 & 10 & 20,8 \\
Palatino & 26 & 22,6 & 16 & 27,6 & 13 & 27,1 \\
Distal & 34 & 29,6 & 14 & 24,2 & 11 & 22,9 \\
Apical & 9 & 7,8 & 4 & 6,8 & 6 & 12,5 \\
Total & 115 & 100 & 58 & 100,00 & 48 & 100,00 \\
\hline
\end{tabular}

$\mathrm{F}=$ Frecuencia. $(\mathrm{n})=$ Número de forámenes. $(\%)=$ Porcentaje.
Además al comparar la probabilidad de emergencia del foramen apical en cada una de las superficies radiculares apicales entre las raíces, no se obtuvo una diferencia significativa $(\mathrm{p} 1=0,371)$ $(p 2=0,670)(p 3=0,577)(p 4=0,549)$ (p5=0,527).

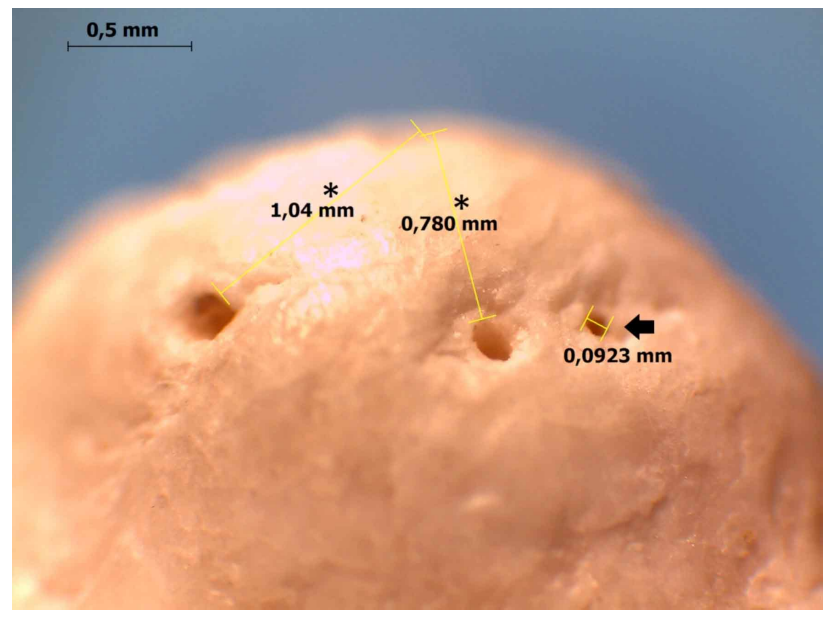

Fig. 2. Microfotografía aumento 4x, Desviación de forámenes apicales mayores. $\left({ }^{*}\right)$ Distancia medida entre foramen apical mayor y ápice anatómico en primer premolar superior. (Flecha) Foramen apical accesorio.
Distancia entre foramen apical mayor y ápice anatómico. El promedio de la distancia medida de todos los forámenes mayores observados (221) desde el punto superior del foramen apical mayor al punto superior del vértice anatómico resultó $0,819 \mathrm{~mm}$. con una desviación estándar de 0,65. Fig. 2.

\section{DISCUSIÓN}

Al analizar el número de forámenes apicales mayores en primeros premolares, se obtuvo resultados similares a los encontrados en el estudio de Ayranci et al. (2013) realizado en población turca, tanto en primeros premolares maxilares unirradiculares en los cuales el resultado obtenido fue una mayor frecuencia de dos forámenes, seguido por un foramen y en primeros premolares maxilares birradiculados, en los cuales se encontró un foramen por raíz. (Ayranci et al.). 
En relación al porcentaje de forámenes apicales mayores en la superficie radicular apical de primeros premolares maxilares unirradiculares, en un estudio realizado por Tian et al. (2012) en la población china, observaron que en dientes con una raíz, una mayor frecuencia la presencia de un foramen con un $64 \%$, seguido por la emergencia de dos forámenes con un $34 \%$. (Tian et al.). Estos resultados no coinciden en proporción con los del presente estudio, pero sí, en la mayor probabilidad de encontrar un foramen, seguido por la presencia de dos forámenes.

Esto es importante de considerar al momento de pesquisar la existencia de uno o dos canales radiculares y la disposición que éstos tienen, emergiendo en uno o dos forámenes, tanto al examen radiográfico convencional como al examen táctil realizado con limas endodónticas.

Como no se encontró una diferencia en el número de forámenes apicales mayores presentes entre las raíces vestibular y palatina de primeros premolares birradiculares, donde en ambos fue la presencia de un foramen, clínicamente podemos deducir que, existe una menor probabilidad de encontrar variabilidad en la anatomía apical de sistema de canales radiculares.

Al analizar el número de forámenes accesorios se obtuvo que existe una gran variabilidad en el número de foraminas presentes en la superficie radicular tanto en los dientes unirradiculares como en las raíces de los dientes birradiculares. Sin embargo, en dientes birradiculares existió un mayor porcentaje de raíces con ausencia de foraminas; por lo que se podría esperar que éstos dientes tengan menor variabilidad en su anatomía, comparado a los premolares unirradiculares. Estos resultados concuerdan con el estudio de Ayranci et al. realizado en población turca, en él cual se estableció que el primer premolar superior unirradiculado, es un diente que presenta una alta prevalencia de foraminas en su superficie radicular apical (Ayranci et al.). El alto número de foraminas en dientes unirradiculares, sugerirían la presencia de canales accesorios y/o deltas apicales, lo que nos indica que son dientes que poseen una anatomía compleja, con una alta prevalencia de ramificaciones en el canal radicular o la presencia de múltiples canales en el ápice, lo que tiene importancia clínica, dificultando un completo desbridamiento de ésta zona, por lo que existe una mayor posibilidad de dejar tejido necrótico y microorganismos en la zona.
En cuanto a la ubicación del foramen apical mayor en la superficie radicular en dientes unirradiculares se encontraron resultados diferentes a los obtenidos en el estudio de Blaskovic'-Subat et al. (1992) realizado en población yugoslava, donde obtuvo que la emergencia del foramen mayor fue en mayor porcentaje por distal (60\%), seguido por mesial (30 \%) (Blaskovic'-Subat et al.). Esto, toma relevancia clínica, ya que, al momento de determinar la longitud de trabajo si se ubica por mesial o distal ésta será posible de visualizar radiográficamente. También tiene una importancia al momento de lograr la permeabilidad del canal, ya que, al conocer ésta mayor casuística, lograremos tomar las medidas necesarias para disminuir así los accidentes clínicos (como precurvar la lima para lograr la permeabilidad).

Respecto a la ubicación del foramen apical en la superficie radicular en dientes birradiculares, a pesar de, presentar mayor frecuencia de emergencia en la superficie radicular palatina, no se puede establecer que sea así en todos los casos, sino que, la posibilidad de que la emergencia del foramen apical mayor es, en cualquiera de las cinco superficies radiculares. Lo anteriormente expresado también se ve reflejado en el estudio de Blaskovic'-Subat et al. en población yugoslava, donde obtuvieron que la ubicación de la emergencia del foramen mayor en primeros premolares superiores birradiculares fue: superficie vestibular (30\%), lingual (30\%), mesial (20\%) y distal (20\%) en la raíz vestibular. En la raíz palatina los resultados obtenidos fueron: por mesial (45 \%) distal (33\%) lingual (11\%) y vestibular (11\%) (Blaskovic'-Subat et al.). Clínicamente éste resultado debe ser considerado, ya que, existe una alta probabilidad que los primeros premolares sean birradiculares, y éstos al ser radiografiados, es difícil la determinación de la emergencia del foramen por la gran superposición de estructuras, dificultando la determinación de la longitud de trabajo en éstos dientes, en donde pueden presentar la ubicación del foramen en cualquier de sus superficies radiculares (Tian et al.).

Acerca de la distancia entre el foramen apical mayor y el ápice anatómico el promedio obtenido se asemeja al de Blastovic-Subat realizado en población yugoslava de 0,99 mm. (Blaskovic'-Subat et al.). Y también con el promedio de 0,67 mm obtenido por Martos et al. (2009), realizado en población brasileña.

Cabe mencionar que en un número no menor de raíces se observó la presencia de canales laterales, lo que puede tener implicancia clínica. Se sugiere realizar un estudio más específico sobre el tema. 
Es relevante destacar que los resultados obtenidos bajo condiciones en que se efectúo éste estudio, no son las mismas que las utilizadas en los estudios nombrados, tal como el número de muestras y etnias, lo que podría explicar los distintos resultados.

\section{AGRADECIMIENTOS}

Agradecemos el asesoramiento y la ayuda en el análisis de los datos al Dr. Benjamín Martínez. También al Dr. Jaime de Calisto por su buena disposición en la revisión del manuscrito.

DOUSSOULIN, G. L.; GALLEGUILLOS, V. C.; GÁLVEZ, B. C. Number and arrangement of the apical foramen in root surface of maxillary first premolars. Int. J. Odontostomat., 10(3):419-424, 2016.

ABSTRACT: The first maxillary premolar tooth has a high variation in its anatomy and morphology. Therefore, knowledge of its anatomy is important at the time of endodontic treatment. The purpose of this study was to describe frequency, number and arrangement of apical foramen in external root apical region in maxillary first premolar. We studied under microscopy (4x) of 141 root of first maxillary premolar single-rooted teeth (51) and double-rooted teeth (49) in Chilean population; where the number of major apical foramen and accessories (foraminas), major foramen location and distance from it to the anatomical apex was observed. In single-rooted teeth the emergence of one or two major foramen was found, averaging 2.5 foramina accessory. In doublerooted teeth the emergence of one foramen was observed with an average of 1.3 foraminas by root. It was established that there were no significant differences in the location of the major foramen between the mesialdistal-palatal surfaces in single-rooted teeth. In doublerooted teeth the major apical foramen was observed in all root surfaces without significant differences between them. The average distance between the major foramen and the anatomic apex foramen was $0.819 \mathrm{~mm}$. In conclusion, the first premolars of Chilean population, have a changeable and complex apical anatomy, in relation to the number of foramina, the number of foramen and its location.

KEY WORDS: apical foramen frequency, accessory foraminas frequency, apical foramen distance to apex, root surface, maxillary first premolar.

\section{REFERENCIAS BIBLIOGRÁFICAS}

Ayranci, L. B.; Yeter, K. Y.; Arslan, H. \& Kseog lu, M. Morphology of apical foramen in permanent molars and premolars in a Turkish population. Acta Odontol. Scand., 71(5):1043-9, 2013.

Blaskovic-Subat, V.; Maricic, B. \& Sutalo, J. Asymmetry of the root canal foramen. Int. Endod. J., 25(3):158-64, 1992.

Chaparro, A. J.; Segura, J. J.; Guerrero, E.; Jiménez-Rubio, A.; Murillo, C. \& Feito, J. J. Number of roots and canals in maxillary first premolars: study of an Andalusian population. Endod. Dent. Traumatol., 15(2):65-7, 1999.

Ferlini-Filho, J. Estudio Radiográfico e Microscópico das Reabsorcoes Radiculares na Presenca de Periodontitesapicais Crónicas. Tese Faculdade de Odontología de Bauru. Sao Paulo, Universidade de Sao Paulo, 1999. pp.186.

Gutiérrez, J. H. \& Aguayo, P. Apical foraminal openings in human teeth. Number and location. Oral Surg. Oral Med. Oral Pathol. Oral Radiol. Endod., 79(6):769-77, 1995.

Kuttler, Y. A precision and biologic root canal filling technic. J. Am. Dent. Assoc., 56(1):38-50, 1958.

Levy, A. B. \& Glatt, L. Deviation of the apical foramen from the radiographic apex. J. N. J. State Dent. Soc., 41(8):12-3, 1970.

Martos, J.; Ferrer-Luque, C. M.; González-Rodríguez, M. P. \& Castro, L. A. Topographical evaluation of the major apical foramen in permanent human teeth. Int. Endod. J., 42(4):329-34, 2009.

Marroquin, B. B.; El-Sayed, M. A. \& Willershausen-Zönnchen, B. Morphology of the physiological foramen: I. Maxillary and mandibular molars. J. Endod., 30(5):321-8, 2004.

Tian, Y. Y.; Guo, B.; Zhang, R.; Yu, X.; Wang, H.; Hu, T. \& Dummer, P. M. Root and canal morphology of maxillary first premolars in a Chinese subpopulation evaluated using cone-beam computed tomography. Int. Endod. J., 45(11):996-1003, 2012.

Dirección para correspondencia:

Dra. Constanza Gálvez B.

Cirujano Dentista

Facultad de Odontología

Universidad Mayor

Santiago

CHILE

Email: conti_gal@hotmail.com

Received: 01-05-2016

Accepted: 24-11-2016 